\title{
Tribune et libres propos
}

\section{(2) OpenEdition}

1 Journals

Édition électronique

URL : http://journals.openedition.org/ries/2813

DOI : 10.4000/ries.2813

ISSN : 2261-4265

Éditeur

Centre international d'études pédagogiques

\section{Édition imprimée}

Date de publication : 1 mars 1999

ISBN : 9771254459005

ISSN : 1254-4590

\section{Référence électronique}

"Tribune et libres propos », Revue internationale d'éducation de Sèvres [En ligne], 21 | mars 1999, mis en ligne le 17 avril 2013, consulté le 08 janvier 2020. URL : http://journals.openedition.org/ries/2813 ; DOI : 10.4000/ries.2813

Ce document a été généré automatiquement le 8 janvier 2020.

(c) Tous droits réservés 


\section{Tribune et libres propos}

1 Au moment de clore le dossier « Formation des enseignants » la rédaction a questionné les participants sur quelques items...

\section{Théorie et pratique}

2 Ces deux termes font problème, plus précisément leur « articulation ». La formation a trois composantes indissociables: une composante intellectuelle, une composante didactique, une composante sociale. Le métier de "maître " se forge dans la prise en compte de ces trois composantes.

Jean-Pierre Bénichou, France

3 Traditionnellement il est convenu que les professeurs du secondaire demandent plus de formation « académique ", cependant que les enseignants du primaire demandent plus de savoir-faire pratique. Dans une approche intégrée vers l'éducation, théorie et pratique devraient se rapprocher l'une vers l'autre.

Anne-Marie Bergh, Afrique du Sud

4 La professionnalisation est un processus qui accompagne la vie d'un enseignant et qui n'est jamais terminé. Les formations initiale et continue servent avant tout à créer un niveau de réflexion et d'exigence qui stimule et soutient cette professionnalisation progressive.

Rainer Gartenschläger, Allemagne

5 Toute pratique doit être solidement enracinée dans une théorie récente et appropriée et la relation entre les deux est quelque chose que l'étudiant doit continuellement explorer.

Carole King, Angleterre

6 Théorie et pratique demeurent disjointes dans la formation, ce qui donne lieu au choc des deux «modèles » incompatibles : un modèle de "réception » des connaissances établies, au niveau théorique, et un modèle "d'appropriation » et de recherche au niveau de la pratique de la classe.

Joseph Giordani, France 


\section{Professionnalisation de l'enseignant}

7 Il existe une distinction entre professionnalisme et professionnalisation. Le premier comporte les valeurs et l'engagement éthique, aussi bien que les concepts, les connaissances, les savoir-faire requis pour la pratique professionnelle. La professionnalisation, d'autre part, tend à augmenter les qualifications et le statut, et peut avoir des conséquences externes.

Anne-Marie Bergh, Afrique du Sud

8 Elle n'est possible que si l'implication du stagiaire dans la vie professionnelle est totale, constante. Il est possible d'organiser la formation de manière à associer les générations de maîtres à l'exercice du métier et de chercher ensemble les solutions qui se posent à des problèmes concrets.

Jean-Pierre Bénichou, France

9 Surtout dans les pays latins, il faut accepter l'idée de la nécessité d'améliorer la professionnalisation des enseignants, même à travers les nouvelles technologies et leur valorisation.

Gianni Colombo, Italie

\section{Rôle de l'université}

10 L'université reste trop dans la dimension disciplinaire. Le développement de modules de préprofessionnalisation en DEUG et licence (ensemble du système éducatif, stages de pratique accompagnée, etc.) est à articuler avec l'IUFM afin de mettre de la cohérence sur cinq ou six années. D'autre part, nécessité de la mise en place d'un entretien aux concours de recrutement pour appréhender la dimension relationnelle.

Daniel Gobert ${ }^{1}$, France

11 L'université dispense les connaissances requises à l'exercice du métier d'enseignant (NB : ce n'est pas la finalité de l'université). Chaque enseignant doit avoir acquis ses savoirs dans un contexte universitaire afin d'être doublement au fait des connaissances inhérentes à sa discipline : fondement et recherche. C'est un problème difficile à régler. Toute tentative pour mettre en place des licences adaptées pour l'enseignement et même les modules de préprofessionnalisation ont été voués à l'échec.

Jean Guglielmi, France.

12 «L'université enseigne des disciplines scientifiques et non des professions », disent les puristes. Mais il faut qu'elle enseigne et intègre les disciplines en vus d'une profession. Voilà ce que l'université doit mieux réussir dans l'avenir de la formation des enseignants qu'elle ne l'a fait dans le passé.

Rainer Gartenschläger, Allemagne.

13 L'éducation supérieure a un rôle unique à jouer comme partenaire dans la formation, en facilitant la recherche et en offrant un espace neutre pour confronter politique et principes.

Muriel Robinson, Angleterre 


\section{Caractéristiques essentielles du formateur d'enseignants}

14 Avoir une vision globale du système éducatif, être capable de travailler sur plusieurs aspects de la profession. La formation saucissonnée (d'un côté le disciplinaire, de l'autre la gestion de la classe, l'orientation et la citoyenneté) ne peut que renforcer(ou déformer d'emblée) la représentation que les enseignants ont de l'enseignement et de leur mission.

Dominique Gelin, France

15 Les équipes devront compter un noyau dur d'enseignants du terrain.

Jean-Pierre Bénichou- Antony - France

16 Posséder une compréhension et une perspective de ce qu'est être enseignant: avoir enseigné dans plusieurs collèges et lycées, avoir étudié les théories des grands pédagogues, philosophes, sociologues, avoir réussi, afin de faire réussir. Pour faire mentir le dicton anglais: "Those who can do, do ; those who can't, teach; and those who can 't teach, teach teachers".

Sonia Rouve, England

17 Bien que beaucoup d'études aient montré qu'il est difficile de définir ces caractéristiques, un certain nombre de capacités minimales sont requises: 1 . une connaissance de la pédagogie et des évolutions dans le domaine des recherches, en étant, si possible, impliqué dans une équipe de recherche; 2 . Une connaissance théorique et pratique du système éducatif et de ses évolutions. 3. Une connaissance des méthodes et techniques d'information et de communication.

Jean Guglielmi, France

18 Qualités humaines: Simplicité et efficacité dans la communication professionnelle; patience, équilibre, ténacité. 2. Bonne connaissance dans tous les domaines d'étude spécifiques. 3. Pragmatisme et capacité de résoudre rapidement les problèmes.

Gianni Colombo- Milano - Italie

19 Une bonne maitrise disciplinaire, une vue globale et transversale du système. Des expériences variées d'enseignement (de la maternelle à l'université). Des capacités de remise en cause, de souplesse d'organisation.

Daniel Gobert, France

\section{Libres propos}

20 Dans des pays comme l'Allemagne ou la France, le poids des administrations est toujours trop grand et une véritable responsabilisation de chaque enseignant est encore loin. Ne faudrait-il pas - sauf erreur de ma part - jeter un coup d'œil du côté du Danemark ou des Pays-Bas?

Rainer Gartenschläger, Allemagne.

21 En dépit des contextes particuliers, il est possible d'identifier des tendances générales. Dans l'ère post-moderne, on s'achemine des caractéristiques universelles vers la diversité. De même, beaucoup de nos réflexions sur les tendances générales de la formation se justifient seulement dans la conception occidentale de la scolarisation et 
de la formation. Le point de vue que j'exprime provient d'Afrique du Sud, un pays où le monde développé rejoint le tiers monde, où extrême richesse et pauvreté se côtoient, quotidiennement, dans la scolarité et la formation. Enfin, le point de vue est africain, un point de vue marqué par les effets du colonialisme.

Anne-Marie Bergh, Afrique du Sud

Les relations dynamiques entre enseignement et recherche, entre formation initiale et continue, entre gouvernement et universités, sont toutes fondamentales pour une bonne pratique et le développement continu de la profession.

Muriel Robinson, Angleterre

La difficulté de former des enseignants pour le XXI ${ }^{\mathrm{e}}$ siècle vient selon moi, de ce que la formation s'exprime essentiellement en termes de contenus et de savoir, alors qu'il faudrait la définir en termes d'attitudes: curiosité, interrogation, appropriation autonome ou aidée des connaissances, recherche, remise en question. Entre formation et enseignement ne devrait exister aucune discontinuité : il importe de susciter chez l'étudiant en formation les attitudes que l'on souhaite lui voir développer chez ses propres élèves. Ce n'est pas encore le cas.

Joseph Giordani, France.

\section{NOTES}

1. Directeur de l'IUFM d'Étiolles. 